\title{
Study protocol: a multi-professional team intervention of physical activity referrals in primary care patients with cardiovascular risk factors - the Dalby lifestyle intervention cohort (DALICO) study
}

Emelie Stenman ${ }^{1 *+}$, Matti E Leijon ${ }^{1 \dagger}$, Susanna Calling ${ }^{1,2}$, Christina Bergmark ${ }^{3}$, Daniel Arvidsson ${ }^{1}$, Ulf-G Gerdtham ${ }^{1,4,5}$, Kristina Sundquist ${ }^{1}$ and Rickard Ekesbo ${ }^{1,3}$

\begin{abstract}
Background: The present study protocol describes the trial design of a primary care intervention cohort study, which examines whether an extended, multi-professional physical activity referral (PAR) intervention is more effective in enhancing and maintaining self-reported physical activity than physical activity prescription in usual care. The study targets patients with newly diagnosed hypertension and/or type 2 diabetes. Secondary outcomes include: need of pharmacological therapy; blood pressure/plasma glucose; physical fitness and anthropometric variables; mental health; health related quality of life; and cost-effectiveness.
\end{abstract}

Methods/Design: The study is designed as a long-term intervention. Three primary care centres are involved in the study, each constituting one of three treatment groups: 1) Intervention group (IG): multi-professional team intervention with PAR, 2) Control group A (CA): physical activity prescription in usual care and 3) Control group B: treatment as usual (retrospective data collection). The intervention is based on self-determination theory and follows the principles of motivational interviewing. The primary outcome, physical activity, is measured with the International Physical Activity Questionnaire (IPAQ) and expressed as metabolic equivalent of task (MET)-minutes per week. Physical fitness is estimated with the 6-minute walk test in IG only. Variables such as health behaviours; health-related quality of life; motivation to change; mental health; demographics and socioeconomic characteristics are assessed with an electronic study questionnaire that submits all data to a patient database, which automatically provides feed-back to the health-care providers on the patients' health status. Cost-effectiveness of the intervention is evaluated continuously and the intermediate outcomes of the intervention are extrapolated by economic modelling.

Discussions: By helping patients to overcome practical, social and cultural obstacles and increase their internal motivation for physical activity we aim to improve their physical health in a long-term perspective. The targeted patients belong to a patient category that is supposed to benefit from increased physical activity in terms of improved physiological values, mental status and quality of life, decreased risk of complications and maybe a decreased need of medication.

\footnotetext{
* Correspondence: emelie.stenman@med.lu.se

${ }^{\dagger}$ Equal contributors

${ }^{1}$ Center for Primary Health Care Research, Lund University, Malmö, Sweden

Full list of author information is available at the end of the article
} 


\section{Background}

The present protocol describes the trial design of a Swedish primary care intervention cohort study, which examines whether an extended, multi-professional physical activity referral (PAR) intervention is more effective in enhancing and maintaining the level of physical activity than physical activity prescription in usual care. The target group is patients with newly diagnosed type 2 diabetes and/or hypertension. The study is designed as a long-term intervention with recurrent analyses of selfreported physical activity levels as well as the effects of physical activity on health-related variables and costeffectiveness.

Hypertension and type 2 diabetes belong to the major risk factors for cardiovascular disease that can be modified by a change in lifestyle, such as increased physical activity [1-3]. Several studies confirm that physical activity reduces the systolic and diastolic blood pressure in patients with hypertension, and improves the glycemic control in subjects with type 2 diabetes $[2,4]$. The evidence suggests that lifestyle modifications and exercise therapy are as effective as pharmacological treatment in selected cases. According to current guidelines, lifestyle modifications should be the first-line treatment alternative for patients with diagnose, or risk for, type 2 diabetes or hypertension [5,6]. Daily or almost daily aerobic physical activity of moderate intensity, such as brisk walking or swimming, for at least 30 minutes is recommended for both conditions [5-7].

\section{The extended PAR intervention}

Written prescriptions of physical activity - in Sweden commonly referred to as PARs - provide an attractive alternative for encouraging patients to increase their physical activity levels and thereby improve health and quality of life. These prescriptions are provided by registered health care professionals in primary care and hospitals $[8,9]$. The effectiveness of Physical activity or exercise referrals and exercise referral schemes show mixed results. A review article shows that physical activity referrals and exercise referrals increase the physical activity levels in certain populations, although the effect tends to wear off over time [10]. A more recent review state that uncertainty remains about the effectiveness of exercise referral schemes for increasing physical activity and improving health [11]. The Swedish Council on Technology Assessment in Health Care, SBU, state that advice and counseling of patients increases physical activity by $12-50 \%$ for at least 6 months, and that a supplementing prescription can increase the activity even further [12]. Clearly, more research is needed to elucidate predictors of successful adherence to this kind of prescriptions or referrals.

Factors that influence the level of physical activity are often classified as individual (e.g. demography, cognitive skills, self-efficacy), interpersonal (e.g. encouragement from family, friends or care givers) or environmental (e.g. culture, physical surroundings, access). Self-efficacy, self-perceived health status and social support belong to the most important predictors of exercise behavior [13]. Other reasons for performing physical activity include improved appearance, enjoyment of the activity itself, social interaction and stress relief $[13,14]$.

To optimize the adherence to PARs and increase the level of physical activity in patients with newly diagnosed type 2 diabetes or hypertension, we will introduce an extended PAR intervention. The aim is to give the patients sufficient support to become regularly active and prevent relapse into inactivity.

The step-based intervention involves a multi-disciplinary primary care team, which may include e.g. physician, hypertension/diabetes nurse, psychologist, physiotherapist or occupational therapist. The team will, in consultation with the patient, customize a PAR based on the summarized results of a pre-test, which includes a computerized study questionnaire, a 6-minute walk test, and physiological status. The study questionnaire is designed to capture factors that may impact the level of physical activity such as self-efficacy and general health behaviors [15]. The multi-disciplinary team will use the principles of SelfDetermination Theory (SDT) and Motivational Interviewing (MI) throughout the patient meetings to improve the adherence to the intervention.

The suggested PAR concept is somewhat different from the original Swedish PAR schemes, which are written prescriptions of individual activities, such as jogging, or group-based activities, such as aerobics, and commonly designed according to the national recommendations for respective disease. In our extended concept, the PAR is based on the patient's preferences and interests, physical and mental status, and motivation.

\section{Self-Determination Theory (SDT)}

SDT is a theory of motivation, which was initially developed by Deci and Ryan [16,17]. SDT focuses on internalization of motivation in order to increase qualities such as perseverance [18], which is crucial for maintenance of profound lifestyle changes. SDT defines 3 innate psychological needs that are crucial for internalization of motivation: autonomy, competence and relatedness $[17,18]$. Satisfaction of these 3 needs improves the internalization of motivation along a continuous scale that extends from amotivation (lack of intention to act), over extrinsic motivation (when the activity is performed to achieve outcomes that are separable from the behaviour itself), to intrinsic motivation (which comes from the satisfactions of the behaviour itself) $[16,17]$ (Figure 1). As can be seen in Figure 1, extrinsic motivation is divided into 4 subgroups: external regulation, introjected regulation, 


\begin{tabular}{|c|c|c|c|c|c|c|}
\hline Type of & Amotivation & & Extrinsic n & notivation & & Intrinsic \\
\hline $\begin{array}{l}\text { Type of } \\
\text { regulation }\end{array}$ & $\begin{array}{l}\text { Non- } \\
\text { regulation }\end{array}$ & $\begin{array}{l}\text { External } \\
\text { regulation }\end{array}$ & $\begin{array}{l}\text { Introjected } \\
\text { regulation }\end{array}$ & $\begin{array}{l}\text { Identified } \\
\text { regulation }\end{array}$ & $\begin{array}{l}\text { Integrated } \\
\text { regulation }\end{array}$ & $\begin{array}{l}\text { Intrinsic } \\
\text { regulation }\end{array}$ \\
\hline $\begin{array}{l}\text { Locus of } \\
\text { causality }\end{array}$ & Impersonal & External & $\begin{array}{l}\text { Somewhat } \\
\text { external }\end{array}$ & $\begin{array}{l}\text { Somewhat } \\
\text { internal }\end{array}$ & Internal & Internal \\
\hline $\begin{array}{l}\text { Degree of } \\
\text { internalization }\end{array}$ & & & & & High & \\
\hline
\end{tabular}

Figure 1 The Self-determination continuum. The figure shows the different types of motivations, regulations and loci of causality according to SDT. One of the aims is to evaluate the patients' degree of internalization at baseline and changes in along the continuum during the course of the study. Adapted from [17,18].

identified regulation and integrated regulation [16,18]. Thus, the patients' motivation can move stepwise within extrinsic motivation.

\section{Motivational Interviewing (MI)}

The 3 innate psychological needs competence, autonomy, and relatedness, defined by SDT, are believed to be promoted by 3 environmental factors: structure, autonomy support and involvement [19]. These 3 environmental factors can in turn be supported by MI. MI is a clientcentred counselling style that aims to elicit behaviour changes by helping clients to explore and resolve ambivalence $[19,20]$ (Figure 2). The general principles of MI have been shown to correspond very well with the intentions of motivational internalization in SDT: whereas SDT provides a theoretical framework for internalization of motivation, MI is more practically oriented and can help to translate the SDT concept into practice $[19,21]$.
The principles of MI have previously been shown to be effective in improving health behaviours such as diet, exercise and addiction treatment [21].

\section{Health economic aspects}

One of the main priorities of Sweden's National Public Health Policy is to increase the level of physical activity in the population and a systematic literature review published by the Swedish Council on Technology Assessment in Health Care points to health economic evaluations of physical activity intervention as one area of special importance in future research. This taken together with the relatively scarce, and of varying quality, health economic studies on interventions promoting physical activity that exists today, makes this field of special importance. Economic evaluation of interventions to improve the management of diabetes have, in recent years, increasingly relied on modelling of long term outcomes and costs of disease [22]. Simulation models in diabetes typically project both life expectancy and the

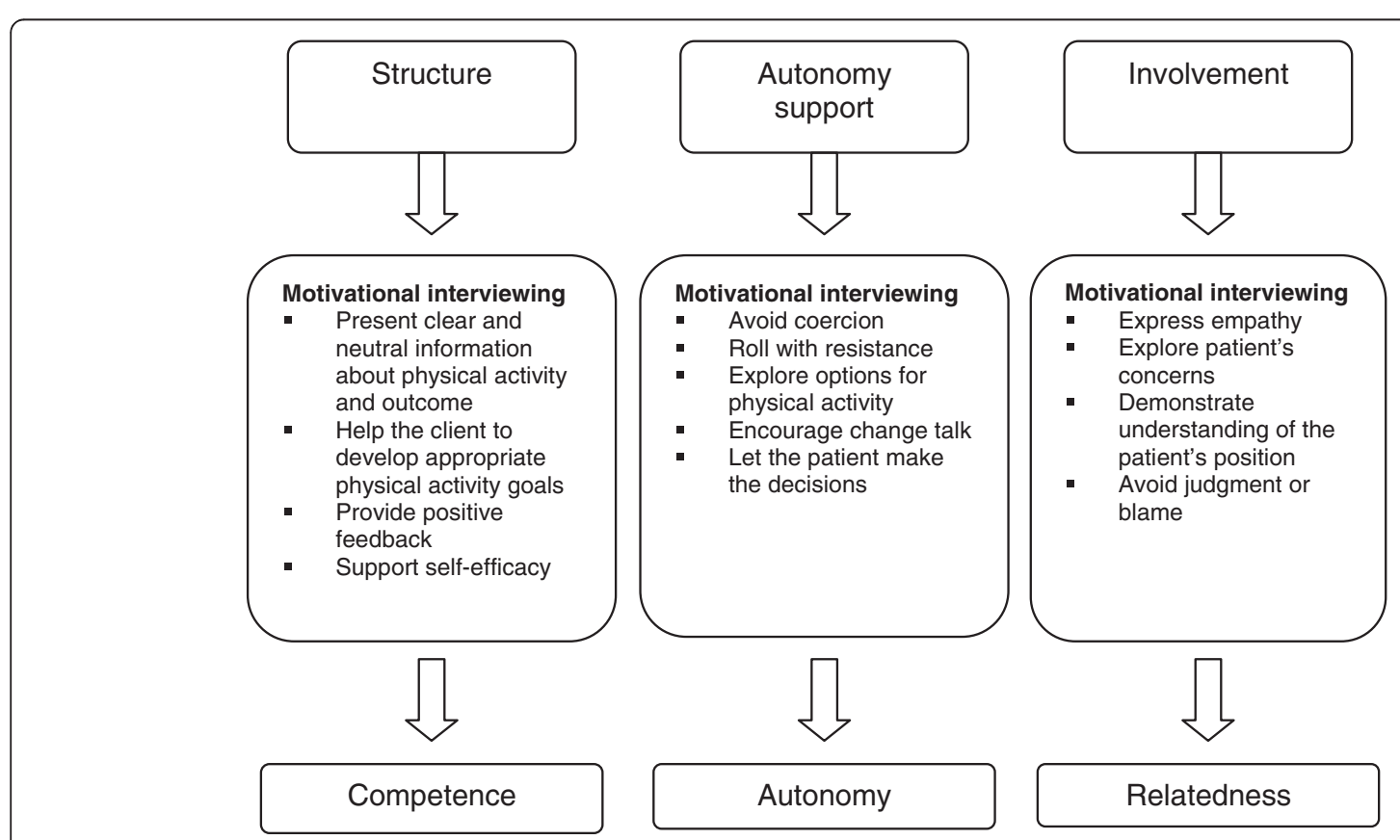

Figure 2 Correlation between Self-determination theory and Motivational Interviewing. Adapted for PAR intervention from [19]. 
degree of morbidity associated with the disease. These estimates can assist in resource planning and can be used to inform economic evaluations, as they quantify potential savings that accrue when interventions reduce the risk of diabetes-related complications. Currently many diabetes simulation models use annual cycles to predict a profile of events over time [23,24]. The benefits of the present intervention are expected to be found in reduced health care and drug utilisation, and increased productivity (measured as reduced sickness absence and increased employment). More specifically, increased physical activity is expected to, among others, reduce the risk of cardiovascular diseases and improve health related quality of life.

\section{Aims}

The first aim of the study is to examine whether an extended multi-professional PAR intervention, based on SDT, is effective in increasing and maintaining the selfreported physical activity level in patients with newly diagnosed type 2 diabetes or hypertension or both.

The second aim is to evaluate changes in a number of health-related variables such as blood pressure, plasma glucose, need of pharmacological therapy, health related quality of life, anxiety and depression, behavioral predictors, physical fitness, obesity, and activities of daily living.

The third aim is to perform an economic evaluation of the intervention from a societal perspective to allow for efficient use of societal resources.

\section{Methods/design}

The study was initiated by Dalby health care centre and involves three primary care centres in Skåne, southern Sweden. Each primary care centre in Sweden has a population of listed patients; the 155 primary care centres in Skåne have between 3000-15500 listed patients and 10-40 employees, e.g. physicians, nurses, physiotherapists, occupational therapists, dieticians, and behavioural scientists.

In the present study, each of the three primary care centers constitutes one of three treatment groups: 1) Intervention group: extended PAR intervention based on a multi-professional team analysis of the study questionnaire and a 6-minute walk test, 2) Control group A: study questionnaire and physical activity prescription in usual care and 3) Control group B: treatment as usual (retrospective data collection). The two control primary care centres have similar socioeconomic and living environment profiles as the Intervention centre. At the health care centres in the Intervention group and Control group A, all patients with newly debuted type 2diabetes or hypertension will be continuously asked to participate by their general practitioners (GPs).
The study is designed as a long-term intervention that will extend over several years with regular analyses of the patients' self-reported physical activity levels, healthrelated variables and cost-effectiveness.

All parts of the study will be conducted according to the principles of the Helsinki Declaration. Written, informed consent will be obtained from all patients entering the Intervention group or Control group A before inclusion.

\section{The 5 A's model}

The study will be performed according to the 5 A's model, which has been the basis for many health behavior change programs [25] and is especially effective for long-term interventions [26]. The model comprises the following 5 steps: Assess; Advice, Agree; Assist; and Arrange, which are continuously followed-up in relation to a personal action plan The contents of each step are presented in Figure 3.

The 5 A's will, in our approach, involve the whole primary care team as well as the patients; different parts of the model will be performed by different team members and some parts may even involve family members or local public health and sports organizations.

\section{Intervention}

A flowchart describing the time course of the study for the Intervention group and Control group A is presented in Figure 4. Below follows a short description of the intervention.

\section{Intervention group}

Eligible patients in the intervention group that consent to take part in the study will go through an extensive intervention program, in which a prescription of physical activity (PAR) will be the central part. At the Baseline visit, the patient meets his/her GP and a nurse. Values for blood pressure (in sitting position after 5 minutes rest; mean of two measurements, supplemented with 24 hour blood pressure monitoring), body mass index (BMI), and abdominal and hip circumference will be taken and a routine heart and lung auscultation and electrocardiography (ECG) will be performed. Furthermore, the patients will fill in an electronic study questionnaire regarding current health status, including variables such as level of physical activity; health behaviours; stress and anxiety; behavioural predictors; health related quality of life; sleeping habits; and activities of daily living ability. The patients will also perform a 6-minute walk test according to a separate, standardized protocol and guided by a physiotherapist. Fasting plasma glucose and other study specific blood samples will be taken in the morning within a few days.

These measures are in accordance with the first and part of the second step of the 5 A's model (Figure 3). 


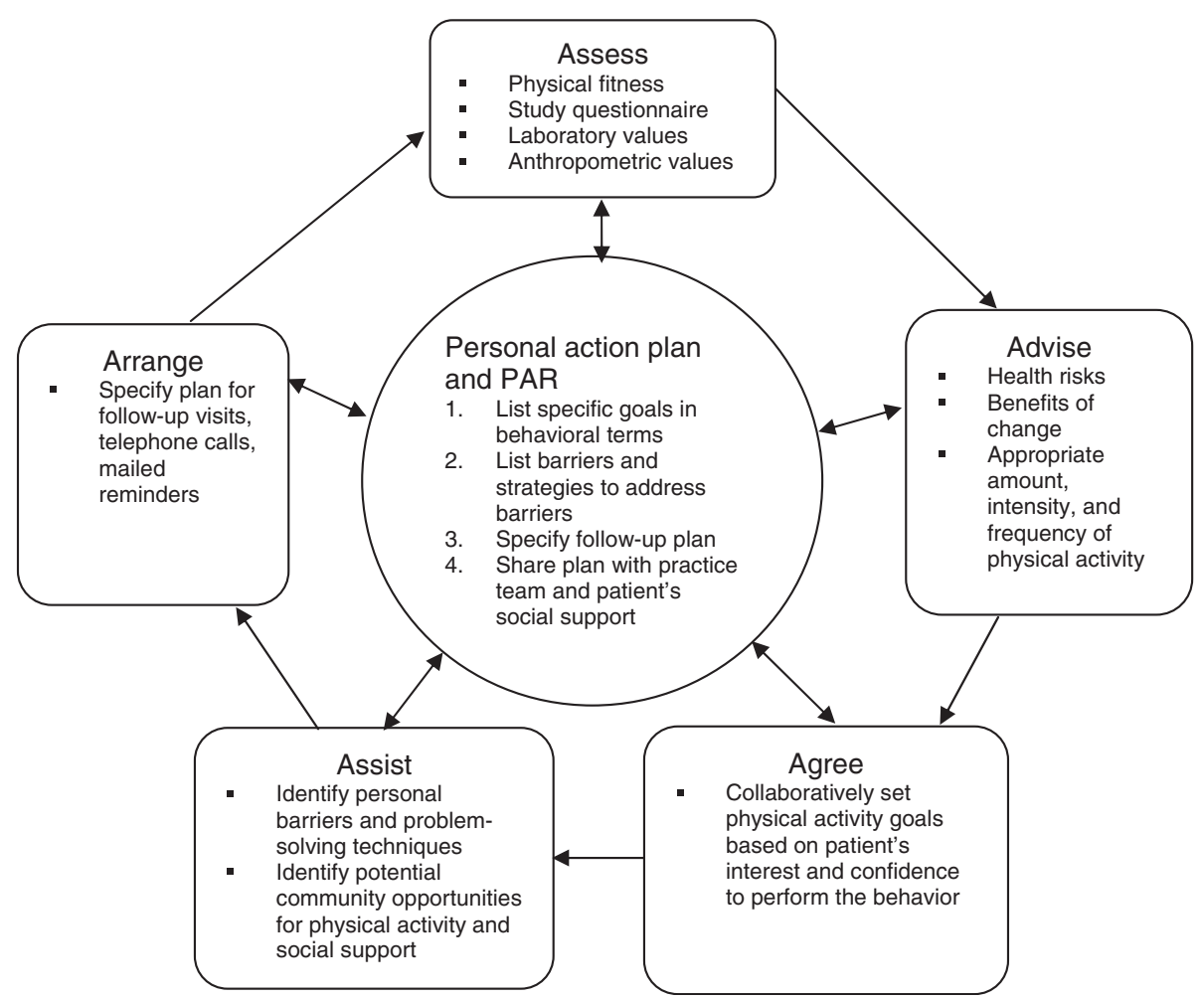

Figure 3 The 5 A's model. Adapted for the extended PAR intervention from $[25,26]$.

After the baseline visit, the patients will be referred to an Initiation visit with one or more members of the multi-professional team comprising psychologist, physiotherapist, occupational therapist, physician and nurse as applicable. The initiation visit will take place within 3 weeks from the baseline visit.

The visit will be based on a team discussion regarding the results of the extended screening including the study questionnaire, the 6-minute walk test and baseline values. It will be structured according to step $2-5$ in the 5 A's model (advise, agree, assist and arrange; Figure 3 ) to provide the patients with a personal action plan based on individual goals and strategies to overcome personal barriers to physical activity. The initiation visit will be founded on the patients' present level of motivation for exercise: amotivation, extrinsic motivation or intrinsic motivation, as described in the SDT model (Figure 1). To improve the patients' autonomy, competence and relatedness regarding a more active lifestyle, the team uses the MI-technique throughout the conversation (Figure 2). It should be noted that all prescribers have attended at least one course in MI. The resulting action plan may include referrals to specialists (psychologist, physiotherapist and/or occupational therapist) for individual consultations and treatments to improve internal motivation and/or physical abilities. The discussion will result in a customized PAR, usually prescribed by a psychologist or physiotherapist from the team together with the patient. The PARs will be tailored to fit the patients' physical and mental condition and motivation, and the recommendations in the Swedish report Physical activity in prevention and treatment of diseases, FYSS, as applicable [27].

\section{Control group $A$}

The patients in Control group A will go through the same physical examinations and study questionnaire as the patients in the Intervention group at the baseline visit. Apart from this, they will get treatment as usual including physical activity prescription as applicable. For Control group A, this is the only study-related visit during the initiation period. They will not attend an additional initiation visit, perform the 6-minute walk test or get a multi-professional team opinion on their treatment.

\section{Follow-up visits}

The patients in the Intervention group and Control group A will return to the clinics for follow-up of the study results regularly (Figure 4). During these follow-up visits, a physical examination, including monitoring of blood pressure and fasting plasma glucose and, if required, a heart- and lung auscultation will be performed. BMI, abdominal and hip circumference will be measured and the patients will fill in the same study 
questionnaire as before or a short version of the study questionnaire, including questions about their activity level. The patients in the Intervention group will also perform regular 6-minute walk tests.

The multi-professional team will, together with the patient, follow up adherence to the physical activity prescription, and modify it if necessary.

\section{Routine visits}

In addition to these study-related visits, the patients will, if necessary, be called to routine visits at the health care centers for blood pressure and plasma glucose checkups regularly.

\section{Control group B}

The patient data in Control group B will be collected from the medical records of patients, diagnosed during the same time period as the other two groups, retrospectively. The same baseline data will be collected as in the other two groups, i.e. time to initiating pharmacological treatment, number of medications and laboratory as well as clinical data. The data will be collected on a population basis. 


\section{Inclusion and exclusion criteria}

The main inclusion criteria are newly diagnosed type 2 diabetes and/or hypertension. Consecutive primary care patients with suspected type 2 diabetes or hypertension will be screened for inclusion. The diagnose criterion for type 2 diabetes in both the Intervention and the Control groups is defined as a $2 \mathrm{x}$ fasting plasma glucose (fPglu) $\geq 7.0 \mathrm{mmol} / \mathrm{l}$ according to WHO guidelines [28]. The diagnose criterion for hypertension is defined as a resting systolic blood pressure $\geq 140 \mathrm{mmHg}$ or a diastolic blood pressure $\geq 90 \mathrm{mmHg}$. Hypertension is confirmed by a 24-hour ambulatory blood pressure monitoring: a systolic blood pressure $\geq 135 \mathrm{mmHg}$ and/or a diastolic blood pressure $\geq 85 \mathrm{mmHg}$ is considered confirmatory of hypertension since ambulatory blood pressure is usually a few $\mathrm{mmHg}$ lower than office blood pressure [5]. If the resting blood pressure exceeds $160 / 100 \mathrm{mmHg}$ at two consecutive measurements within one week, the patient will be prescribed pharmacological treatment in addition to physical activity. Patients with a history of type 2 diabetes or hypertension that get diagnosed by the other diagnose will be included in the study as a subgroup, in which a reduction of current medication is one endpoint. On the other hand, patients with a history of type 1 diabetes will be excluded from the study. The overall inclusion and exclusion criteria of the study are presented in Table 1.

\section{Outcome measures}

\section{Primary outcome}

Level of self-reported physical activity (as assessed with the International Physical Activity Questionnaire (IPAQ) and expressed as MET-minutes).

\section{Secondary outcomes}

(1) Need of pharmacological therapy for hypertension or type 2 diabetes; (2) Blood pressure/plasma glucose; (3) Physical fitness and anthropometric variables; (4) Mental health (anxiety, depression, sleep, stress); (5) Health related quality of life; and (6) Cost-effectiveness of intervention.

\section{Assessment of physical activity level (primary outcome)} Individual physical activity levels will be assessed with the International Physical Activity Questionnaire (IPAQ), a validated instrument for assessing physical activity [30], in the Intervention group and Control group A. IPAQ comprises 4 simple questions on physical activity, which will be included in the study questionnaire. The IPAQ results will be expressed as MET-minutes per week. A metabolic equivalent, or MET, is a unit that describes the energy expenditure of a specific activity. A MET is the ratio of the rate of energy expended during a specific activity to the resting metabolic rate. $1 \mathrm{MET}$ is equivalent to the resting metabolic rate, while a 2 MET-activity requires two times the metabolic energy expenditure of sitting quietly [31]. If a person does a 2 MET-activity for 30 minutes, he or she has done $2 \times 30=60$ MET-minutes of physical activity.

\section{Assessment of physical fitness and anthropometric variables (secondary outcome)}

Physical fitness will be estimated with the 6-minute walk test in the Intervention group only. The 6-minute walk test measures the distance that a patient can walk on a flat, hard surface in 6 minutes, usually on a short indoor track. This test is useful as a complement to the subjective assessment in the study questionnaire since it

Table 1 Overall inclusion and exclusion criteria

\begin{tabular}{|c|c|c|}
\hline A & \multicolumn{2}{|l|}{ Overall inclusion criteria } \\
\hline & \multicolumn{2}{|c|}{ 1. Newly diagnosed type 2 diabetes (a) or hypertension (b) or both } \\
\hline & a) Type 2 diabetes & b) Hypertension \\
\hline & $2 \times$ fasting plasma glucose $(\mathrm{fP}-\mathrm{glu}) \geq 7.0 \mathrm{mmol} / \mathrm{l}$ & $\begin{array}{l}\text { A systolic blood pressure } \geq 140 \mathrm{mmHg} \text { and/or a diastolic bloodpressure } \geq 90 \mathrm{mmHg} \text {, } \\
\text { which isconfirmed by a systolic bloodpressure } \geq 135 \mathrm{mmHg} \text { or a-diastolic blood pressure } \\
\geq 85 \mathrm{mmHg} \text { during a } 24 \text {-hourambulatory monitoring }\end{array}$ \\
\hline & \multicolumn{2}{|l|}{ 2. Male and female patients $\geq 18$ years } \\
\hline \multirow[t]{8}{*}{ B } & \multicolumn{2}{|l|}{ Overall exclusion criteria } \\
\hline & \multicolumn{2}{|l|}{ 1. $<18$ years } \\
\hline & \multicolumn{2}{|l|}{ 2. History of type 1 diabetes } \\
\hline & \multicolumn{2}{|l|}{ 3. Blood pressure > 180/105 mmHg [29] } \\
\hline & \multicolumn{2}{|l|}{ 3. Known pregnancy } \\
\hline & \multicolumn{2}{|c|}{ 5. Cardio- or cerebrovascular event that required hospitalization within the past 3 months } \\
\hline & \multicolumn{2}{|l|}{ 6. Current participation in other clinical study } \\
\hline & 7. Severe mental disorder or substance abuse & \\
\hline
\end{tabular}


evaluates the integrated responses of all body systems that are involved in physical activity including the pulmonary and cardiovascular systems, systemic and peripheral circulation, blood, neuromuscular units and muscle metabolism [32]. In the present study, the 6-minute walk test will be used to evaluate changes in physical fitness expressed as percentage of the individual result at baseline. The patients will perform a "testwalk" for 1 minute before the 6-minute walk test and the test will be performed according to a separate, standardized protocol [32].

Anthropometric measurements will include weight, length, body mass index and waist-to-hip ratio.

\section{Assessment of behavioural predictors, mental health and health related quality of life (secondary outcome)}

With the study questionnaire, psychological variables will be collected to assess a) behavioural predictors, b) mental health and c) health related quality of life. These variables include: a) self-perceived health, self efficacy, self-determination; b) anxiety and depression, stress, panic-related physical sensations, and sleeping quality; c) health related quality of life (Table 2). Almost all these items will be evaluated with questions from already validated questionnaires, which will be included in the study questionnaire. If a person in Control group A gets results that indicates severe depression or anxiety, this person will be offered a medical appointment.

\section{Assessment of cost effectiveness of intervention (secondary outcome)}

The Intervention group will be compared with the two control groups in order to perform cost-effectiveness analysis (CEA) or cost-utility analysis (CUA) or both. All the costs related to the intervention such as training of the personnel and participants' time and travel costs will be counted. Productivity losses (including informal care) and benefits will be counted using appropriate methods such as the friction cost and the human capital approach $[37,38]$. The project in general and the study questionnaire in particular, will be designed to effectively capture the benefits of the intervention, for example, the EQ-5D instrument will be used to examine changes in health related quality of life (http://www.euroqol.org). The intermediate outcomes of the intervention (e.g. reduction of cardiovascular risk factors) will be extrapolated by economic modelling (either Decision tree or Markov model) to predict the long term health related benefits [39,40]. Generating estimates of acute and long-term costs associated with the management of diabetes requires information on the annual average health care costs with a wide spectrum of complications. The most significant costs associated with many complications are likely to be those arising from inpatient hospital episodes. These costs have been estimated for selected countries, for example Sweden [41].

\section{Electronic study questionnaire}

The study questionnaire will be filled in directly on a computer at the health care centre under supervision of

Table 2 Study questionnaire variables with references

\begin{tabular}{|c|c|}
\hline Variable & Reference \\
\hline $\begin{array}{l}\text { Demographic and socioeconomic characteristics and other general } \\
\text { questions concerning background and health related issues like general } \\
\text { health, current medications, symptoms, pain, stress, and sleeping quality. }\end{array}$ & $\begin{array}{c}\text { The Public Health Survey in Skåne, Sweden and questions from the } \\
\text { Swedish Annual Level of Living Survey (SALLS). }\end{array}$ \\
\hline Health related quality of life & EQ-5D (http://www.euroqol.org/) \\
\hline $\begin{array}{l}\text { Lifestyle related habits - general questions related to the individuals: } \\
\text { physical activity, healthy eating, tobacco and alcohol usage }\end{array}$ & The National Board of Health and Welfares Questionnaire. \\
\hline Physical activity level & International Physical Activity Questionnaire (IPAQ) - short version [30]. \\
\hline Stages of change (readiness for change to be more physically active) & Used in previous study [9] \\
\hline Physical activity history and dog ownership and dog-walking & Study specific question \\
\hline Self-efficacy & General Self-Efficacy Scale (http://www.ralfschwarzer.de/) \\
\hline Self-determination & $\begin{array}{l}\text { Perceived Competence Scale (PCS), Health Care Climate Questionnaire } \\
\text { (HCCQ), Treatment Self-Regulation Questionnaire (TSRQ) from the Health- } \\
\text { Care, Self-Determination Theory Packet (http://www.psych.rochester.edu/ } \\
\text { SDT/measures/hc_description.php) }\end{array}$ \\
\hline Anxiety and depression & Hospital Anxiety and Depression (HAD) Scale [33] \\
\hline Anxiety related physical sensations & Anxiety Sensitivity Index (ASI) [34] \\
\hline Activities of daily living (ADL) ability & Canadian Occupational Performance Measure (COPM) [35] \\
\hline - only patients that score $2-3 p$ in question $A, B$, or $C$ in EQ-5D & \\
\hline Non-adherence and reasons for non-adherence to PARs intervention & Used in previous study [36] \\
\hline
\end{tabular}


a health care professional. All data are then submitted to a patient database, which automatically provides instant feed-back on the patients' health status. The study questionnaire comprises questions from validated question batteries, questions from the regional population survey, and some questions specifically designed for the study (Table 2), in order to thoroughly evaluate the patients' health status as well as the study methodology. A shortversion of the questionnaire will be used at some of the follow-up visits (see Figure 4).

\section{Laboratory values}

A study specific blood sample, drawn after an overnight fast, and a morning urine sample will be taken at all study-specific visits to measure lipid profile, plasma glucose, HbA1c, haemoglobin, electrolyte status, thyroid status, high-sensitive C-reactive protein and urinary albumin-to-creatinine ratio. The samples will be analyzed at the Department of Clinical Chemistry, Skåne University Hospital. One frozen blood sample will be collected for long-term storage for potential future analysis.

\section{End of study}

The study, including regular data collection, is designed as a longitudinal intervention that will last over years. The patients will be invited to participate in the intervention until further notice or until they choose to finish their participation themselves (not equivalent to nonadherence to intervention).

The intervention may be extended to evaluate other endpoints than the ones described in the present paper. In that case, ethical approval will be applied for separately.

\section{Adverse events}

At the follow-up visits, the patients will be asked if they have experienced any adverse events since the last visit. All adverse events will be reported, independent of possible relation to the intervention.

\section{Statistical analysis and power calculation}

The first statistical analyses and compilation of results will be performed 18 months after inclusion of the first patient or when the Intervention group and Control group A have included and followed up 60 and 30 eligible patients respectively for at least six months. At that point, data will also be collected retrospectively in control group B. Reasons for non-attendance in the study will be analyzed as well as reasons for non-adherence to the intervention. Allocation will be concealed, and study data blinded, for the bio-statisticians and researchers who will perform the analyses.

The sample size is based on an assumed statistical power of $80 \%$, a significance level of $5 \%$, a difference of 200 MET-minutes between the groups, a standard deviation of 300 MET-minutes and a worst-case loss to follow-up of $30 \%$.

The secondary outcomes blood pressure, plasma glucose, need of pharmacological therapy, and cost-effectiveness will be compared between all three study groups.

\section{Approval and registration}

The study was approved by Lund regional ethical review board, registration number 2010/330, and registered at ClinicalTrials.gov, registration number NCT01187576.

\section{Discussion}

The targeted patients - individuals with newly diagnosed type 2 diabetes or hypertension - belong to a patient category that is indeed supposed to benefit from increased physical activity in terms of improved health variables, decreased risk of complications [27], and possibly also a decreased need of pharmacological therapy [42]. In the present study we combine a patient-centered lifestyle modification by a multi-professional team; a theory-based approach for self-regulation and behavior change; a feedback system for immediate response to treatment and adherence to treatment; and determination of cost-effectiveness of the intervention. This is in agreement with current guidelines for the prevention and management of the targeted diseases [5,6], and may increase the efficiency of treatment and evaluation of intervention.

The intervention intends to increase the patients' internal motivation for physical activity by following the principles of SDT and MI, and thereby improve their adherence to PAR. The ultimate goal is to decrease cardiovascular complications, improve quality of life and work ability and decrease the need of medication. Our hypothesis is that the proposed PAR intervention is a more effective way to support patients in achieving and maintaining a beneficial level of physical activity than treatment as usual. We also believe that this structured intervention can be cost effective if it leads to a better health status and, subsequently, a less need of medical care among these patients.

The reason for using two control groups is that we believe there is a risk that the patients in Control group A may get an increased awareness of physical activity and subsequent alteration of habits, just by participating and filling in the questionnaires. Therefore we are also making a retrospective analysis at a third centre.

The study has some limitations. One possible limitation is the uncertain generalizability of the findings if the results of the intervention are related to individual qualities of the persons delivering the intervention, such as a special expertise and enthusiasm. Other possible limitations of lifestyle intervention studies in general and physical activity interventions in particular are the 
difficulties to determine relevant differences between the groups for the power calculation. The assumed difference of 200 MET-minutes is an ambitious goal and a balance between assumed effect of intervention, time to follow-up, and assumed number of patients. The primary outcome may not turn out significant at the first analysis (after one year). We believe, however, that 200 MET-minutes is a reachable difference and that the difference between the groups will increase over time.

If the extended PAR intervention proves to be successful, the model may be adopted by other primary care centers as well. Our goal is to find an attractive, userfriendly and cost effective PAR scheme, which may be translated to a broad range of patient groups that benefit from increased physical activity.

\section{Abbreviations \\ ADL: Activities of daily living; BMI: Body mass index; CA: Control group A; CB: Control group B; ECG: Electro cardiography; fP-glu: Fasting plasma glucose; IG: Intervention group; IPAQ: International physical activity questionnaire; MET: Metabolic equivalent; MI: Motivational interviewing; PAR: Physical activity referral; SDT: Self-determination theory.}

\section{Competing interests}

The authors declare that they have no competing interests.

\section{Authors' contributions}

RE (MD, PhD and head of Dalby Health Care Centre) introduced the idea and initiated this study. Research Coordinator and Biologist ES (PhD), Research Coordinator Public Health specialist ML (PhD), Psychologist CB, Public Health Nutritionist DA (PhD) and the Family Physicians KS (MD, PhD), SC (MD, PhD), and RE formulated the aim of the study and set up the study logistics. ES drafted the present study protocol. ML coordinated and drafted the study questionnaire. Health Economist UG (PhD) introduced the health economic aspects of the project. All authors contributed to developing the project and all authors read, commented and approved the final version of the manuscript.

\section{Acknowledgements}

We would like to thank Occupational Therapist Monica Berglin Olsson and Physiotherapist Helena Tadic at Dalby Health Care Centre, Biostatistician Karolina Carlsson, Database Manager Tatiana Nyström, and Systems Engineer Christian Andersson at Center for Primary Health Care Research. We would also like to thank Johan Jarl, PhD, and Sanjib Saha, PhD, for input on health economics.

Furthermore, we would like to thank the Swedish Council for Working Life and Social Research (registration number 2007-1754) for economical support.

\section{Author details}

'Center for Primary Health Care Research, Lund University, Malmö, Sweden. ${ }^{2}$ Bara Health Care Centre, Skåne County Council, Skåne, Sweden. ${ }^{3}$ Dalby Health Care Centre, Skåne County Council, Skåne, Sweden. ${ }^{4}$ Department of Economics, Lund University, Malmö, Sweden. ${ }^{5}$ Health Economics \& Management, Institute of Economic Research, Lund University, Malmö, Sweden.

Received: 27 August 2010 Accepted: 31 May 2012

Published: 22 June 2012

\section{References}

1. Fact sheet No 317, Cardiovascular diseases (CVDS). World Health Organization; 2009.

2. Boule NG, Haddad E, Kenny GP, Wells GA, Sigal RJ: Effects of exercise on glycemic control and body mass in type 2 diabetes mellitus: a metaanalysis of controlled clinical trials. JAMA 2001, 286(10):1218-1227.
3. Guidry MA, Blanchard BE, Thompson PD, Maresh CM, Seip RL, Taylor AL, Pescatello LS: The influence of short and long duration on the blood pressure response to an acute bout of dynamic exercise. Am Heart J 2006, 151(6):1312-1325.

4. Cornelissen VA, Fagard RH: Effects of endurance training on blood pressure, blood pressure-regulating mechanisms, and cardiovascular risk factors. Hypertension 2005, 46(4):667-675.

5. Mancia G, De Backer G, Dominiczak A, Cifkova R, Fagard R, Germano G, Grassi G, Heagerty AM, Kjeldsen SE, Laurent S, et al: 2007 Guidelines for the Management of Arterial Hypertension: The Task Force for the Management of Arterial Hypertension of the European Society of Hypertension (ESH) and of the European Society of Cardiology (ESC). $J$ Hypertens 2007, 25(6):1105-1187.

6. Paulweber B, Valensi P, Lindstrom J, Lalic NM, Greaves CJ, McKee M, Kissimova-Skarbek K, Liatis S, Cosson E, Szendroedi J, et al: A European evidence-based guideline for the prevention of type 2 diabetes. Horm Metab Res, 42(Suppl 1):S3-36.

7. In Physical activity guidelines advisory committee report, 2008. Edited by: Washington DUSDohahs: 2008.

8. Leijon ME, Bendtsen P, Nilsen P, Festin K, Stahle A: Does a physical activity referral scheme improve the physical activity among routine primary health care patients? Scand J Med Sci Sports 2009, 19(5):627-636.

9. Kallings LV, Leijon M, Hellenius ML, Stahle A: Physical activity on prescription in primary health care: a follow-up of physical activity level and quality of life. Scand J Med Sci Sports 2008, 18(2):154-161.

10. Morgan O: Approaches to increase physical activity: reviewing the evidence for exercise-referral schemes. Public Health 2005, 119(5):361-370.

11. Pavey TG, Taylor AH, Fox KR, Hillsdon M, Anokye N, Campbell JL, Foster C, Green C, Moxham T, Mutrie N, et al: Effect of exercise referral schemes in primary care on physical activity and improving health outcomes: systematic review and meta-analysis. BMJ 2011, 343:d6462.

12. The Swedish Council on Technology Assessment in Health Care S: Methods of promoting physical activity. In A systematic review. Edited by Statens beredning för medicinsk utvärdering S; 2007.

13. Sherwood NE, Jeffery RW: The behavioral determinants of exercise: implications for physical activity interventions. Annu Rev Nutr 2000, 20:21-44.

14. Befolkningens motivation og barrierer for fysisk aktivitet. In The population's motivation and barriers to physical activity. Edited by Sundhedstyrelsen D; 2003.

15. Silva MN, Vieira PN, Coutinho SR, Minderico CS, Matos MG, Sardinha LB, Teixeir PJ: Using self-determination theory to promote physical activity and weight control: a randomized controlled trial in women. J Behav Med 2009, Epub ahead of print.

16. Ryan RM, Deci EL: Self-determination theory and the facilitation of intrinsic motivation, social development, and well-being. Am Psychol 2000, 55(1):68-78

17. Deci EL, Ryan RM: The "what" and "why" of goal pursuits: human needs and the self-determination of behavior. Psychol Inq 2000, 11(4):227-268.

18. Silva MN, Markland D, Minderico CS, Vieira PN, Castro MM, Coutinho SR, Santos TC, Matos MG, Sardinha LB, Teixeira PJ: A randomized controlled trial to evaluate self-determination theory for exercise adherence and weight control: rationale and intervention description. BMC Publ Health 2008, 8:234.

19. Markland D, Ryan RM, Tobin VJ, Rollnick S: Motivational intervieing and self-determination theory. J Soc Clin Psychol 2005, 24(6):811-831.

20. Miller WR: Motivational interviewing: research, practice, and puzzles. Addict Behav 1996, 21(6):835-842.

21. Vansteenkiste M, Sheldon KM: There's nothing more practical than a good theory: integrating motivational interviewing and self-determination theory. Br J Clin Psychol 2006, 45(Pt 1):63-82.

22. Guidelines for computer modeling of diabetes and its complications. Diabetes Care 2004, 27(9):2262-2265.

23. Clarke PM, Gray AM, Briggs A, Farmer AJ, Fenn P, Stevens RJ, Matthews DR, Stratton IM, Holman RR: A model to estimate the lifetime health outcomes of patients with type 2 diabetes: the United Kingdom Prospective Diabetes Study (UKPDS) Outcomes Model (UKPDS no. 68). Diabetologia 2004, 47(10):1747-1759.

24. Palmer AJ, Roze S, Valentine WJ, Minshall ME, Foos V, Lurati FM, Lammert M, Spinas GA: The CORE Diabetes Model: Projecting long-term clinical outcomes, costs and cost-effectiveness of interventions in diabetes 
mellitus (types 1 and 2) to support clinical and reimbursement decisionmaking. Curr Med Res Opin 2004, 20(Suppl 1):S5-26.

25. Whitlock EP, Orleans $C T$, Pender N, Allan J: Evaluating primary care behavioral counseling interventions: an evidence-based approach. Am J Prev Med 2002, 22(4):267-284.

26. Glasgow RE, Goldstein MG, Ockene JK, Pronk NP: Translating what we have learned into practice. Principles and hypotheses for interventions addressing multiple behaviors in primary care. Am J Prev Med 2004, 27(2 Suppl):88-101.

27. FYSS: FYSS 2008. Fysisk aktivitet i sjukdomsprevention och sjukdomsbehandling [Physical activity in prevention and treatment of diseases]. In The Swedish National Institute of Public Health and Yrkesföreningar för Fysisk Aktivitet. Edited by Health TSNIoP; 2008

28. In Definition and diagnosis of diabetes mellitus and intermediate hyperglycemia. Edited by Organization WH; 2006.

29. American College of Sports Medicine: Position Stand. Physical activity, physical fitness, and hypertension. Med Sci Sports Exerc 1993, 25(10):i-X.

30. Craig CL, Marshall AL, Sjostrom M, Bauman AE, Booth ML, Ainsworth BE, Pratt M, Ekelund U, Yngve A, Sallis JF, et al: International physical activity questionnaire: 12-country reliability and validity. Med Sci Sports Exerc 2003, 35(8):1381-1395.

31. Ainsworth BE, Haskell WL, Leon AS, Jacobs DR Jr, Montoye HJ, Sallis JF, Paffenbarger RS Jr: Compendium of physical activities: classification of energy costs of human physical activities. Med Sci Sports Exerc 1993, 25(1):71-80

32. ATS: Guidelines for the six-minute walk test. Am J Respir Crit Care Med 2002, 166(1):111-117.

33. Zigmond AS, Snaith RP: The hospital anxiety and depression scale. Acta Psychiatr Scand 1983, 67(6):361-370.

34. Reiss S, Peterson RA, Gursky DM, McNally RJ: Anxiety sensitivity, anxiety frequency and the prediction of fearfulness. Behav Res Ther 1986, 24(1):1-8.

35. Law M, Baptiste S, McColl M, Opzoomer A, Polatajko H, Pollock N: The Canadian occupational performance measure: an outcome measure for occupational therapy. Can J Occup Ther 1990, 57(2):82-87.

36. Leijon ME, Bendtsen P, Stahle A, Ekberg K, Festin K, Nilsen P: Factors associated with patients self-reported adherence to prescribed physical activity in routine primary health care. BMC Fam Pract, 11:38.

37. Koopmanschap MA, Rutten FF, van Ineveld BM, van Roijen L: The friction cost method for measuring indirect costs of disease. J Health Econ 1995, 14(2):171-189.

38. Single E, Collins D, Easton B, Harwood H, Lapsley H, Kopp P, Wilson E: International guidelines for estimating the costs of substance abuse. 2nd edition. Geneva: Edited by Organisation WH; 2003.

39. Cobiac $L$, Vos T, Barendregt JJ: Cost-effectiveness of interventions to promote physical activity: a modelling study. PLoS Med 2009, 6(7):e1000110

40. Trikalinos TA, Siebert U, Lau J: Decision-analytic modeling to evaluate benefits and harms of medical tests: uses and limitations. Med Decis Making 2009, 29(5):E22-29.

41. Gerdtham UG, Clarke P, Hayes A, Gudbjornsdottir S: Estimating the cost of diabetes mellitus-related events from inpatient admissions in Sweden using administrative hospitalization data. Pharmacoeconomics 2009, 27(1):81-90.

42. Pedersen BK, Saltin B: Evidence for prescribing exercise as therapy in chronic disease. Scand J Med Sci Sports 2006, 16(Suppl 1):3-63.

doi:10.1186/1472-6963-12-173

Cite this article as: Stenman et al: Study protocol: a multi-professional team intervention of physical activity referrals in primary care patients with cardiovascular risk factors - the Dalby lifestyle intervention cohort (DALICO) study. BMC Health Services Research 2012 12:173.

\section{Submit your next manuscript to BioMed Central and take full advantage of:}

- Convenient online submission

- Thorough peer review

- No space constraints or color figure charges

- Immediate publication on acceptance

- Inclusion in PubMed, CAS, Scopus and Google Scholar

- Research which is freely available for redistribution

Submit your manuscript at www.biomedcentral.com/submit
Biomed Central 